\title{
REPRESENTACIÓN DEL ROL PROFESIONAL EN ASPIRANTES A LA CARRERA DE MEDICINA. FACULTAD DE CIENCIAS MÉDICAS. UNIVERSIDAD NACIONAL DE CÓRDOBA.
}

Carmen Stabile ${ }^{1}$ Alicia Ruth Fernández ${ }^{2}$

${ }^{1}$ Lic. en Psicología. Dra. En Ciencias de la Salud. Docente titular de la Cátedra de Orientación Vocacional y Ocupacional. Universidad Nacional de Córdoba. Facultad de Psicología contacto principal email: carmenstabile@unc.edu.ar ${ }^{2}$ Prof. Dra. en Medicina y Cirugía. Asesora en Investigación. Universidad Nacional de Córdoba. Facultad de Ciencias Médicas. Escuela de Salud Pública y Ambiente

DOI: https://doi. org/10.31052/1853.1180. v24n1

@Universidad Nacional de Córdoba

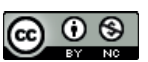

Trabajo recibido: 11 de diciembre 2019. Aprobado: 04 de mayo 2020.
PROFESSIONAL ROLE REPRESENTATION IN APPLICANTS TO MEDICAL STUDIES AT THE SCHOOL OF MEDICAL SCIENCES. NATIONAL UNIVERSITY OF CORDOBA.

REPRESENTAÇÃO DO PAPEL PROFISSIONAL EM CANDIDATOS À CARREIRA DE MEDICINA. FACULDADE DE CIÊNCIAS MÉDICAS. UNIVERSIDADE NACIONAL DE CORDOBA.

\section{Resumen}

Introducción: Los estudiantes que inician una carrera universitaria cuentan con representaciones acerca de la carrera elegida y de su futuro profesional. Objetivo: Identificar la representaciones de los estudiantes preinscriptos de la carrera de Medicina con respecto a la imagen del rol profesional. Metodología: Estudio observacional y transversal, a partir de una encuesta implementada a 1857 estudiantes. Análisis de los datos: ANOVA o Datos Categorizados, con un 95\% de confianza. Resultados: En

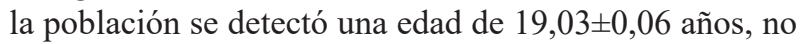
inserta en el mercado laboral, una mayor frecuencia de padres con estudios universitarios. El área elegida para el desempeño del rol es la de "Especialidades Médicas". Los estudiantes encuestados refirieron en cuanto a la imagen del rol profesional en primer lugar, las cualidades personales, la formación permanente y la responsabilidad. Predomina el deseo de ayudar y curar, a través de un rol profesional idóneo. En cuanto a los aspectos preventi- 
vos de la carrera como futuro profesional, el $48 \%$ enuncian el compromiso social y ayudar a la gente, reafirmando el aspecto social y solidario del rol profesional. El $50 \%$ proyecta la idoneidad profesional y continuar con la formación permanente, enfatizando el aspecto cognitivo. Conclusión: La población en estudio, es una población joven, no inserta en el mercado laboral, que no muestra relación entre su elección y la especialidad del secundario que ha cursado, son estimulados por sus progenitores quienes les generan y alientan interés por realizar estudios superiores y muestran, la idoneidad con alto compromiso y solidaridad social, como expectativa profesional.

Palabras clave: Valores sociales- Estudiante - Orientación Vocacional.

\section{Abstract}

Introduction: Students starting university have representations regarding the chosen field of studies and their professional future. Objective: To identify the representations that preregistered students of medicine have, regarding the image of the professional role. Method: Cross-sectional, observational study based on a survey performed to 1857 students. Data analysis: ANOVA or Categorized Data, with 95\% confidence. Results: Age detected in the population was $19.03 \pm 0,06$ years, not working, with higher frequency of parents with university studies. The selected area for role performance is "Medical Specialties". Surveyed students answered regarding the image of the professional role in the first place, personal qualities, ongoing training and responsibility. Willingness to help and cure prevails, through an appropriate professional role. Regarding preventive aspects of the university years as professional future, $48 \%$ mention social commitment and helping people, thus restating the social and supportive aspects of the Professional Role. Fifty percent of the students highlight professional aptitude and ongoing training, emphasizing the cognitive aspect. Conclusion: The studied population is young, does not work, does not show a relationship between their selection and the specialization of their high school studies, is motivated by their parents who develop and boost the interest for university studies and refer to appropriateness and aptitude with high commitment and social solidarity as professional expectations.

Key words: Social values - Students - Career Orientation.

\section{Resumo}

Introdução: Os estudantes que iniciam uma carreira universitária têm representações sobre a carreira escolhida e o futuro profissional. Objetivo: Identificar as representações dos alunos pré-inscritos na carreira de Medicina em relação à imagem do papel profissional. Metodologia: Estudo observacional e transversal, com base em uma pesquisa com 1.857 estudantes. Análise dos dados: ANOVA ou Dados Categorizados, com 95\% de confiança. Resultados: Nessa amostra, detectou-se uma idade de 19,03 $\pm 0,06$ anos, não inserida no mercado trabalhista, uma maior frequência de pais com estudos universitários. A área escolhida para o desempenho do papel é "Especialidades Médicas". Os alunos pesquisados se referiram à imagem do papel profissional em primeiro lugar, as qualidades pessoais, o treinamento contínuo e a responsabilidade. O desejo de ajudar e curar tem predomínio, através de um desempenho profissional idôneo. Quanto aos aspectos preventivos da carreira como futuro profissional, $48 \%$ afirmam o compromisso social e ajudar as pessoas, fazendo destaque do aspecto social e solidário do Papel Profissional. 50\% projetam a aptidão profissional e continuar com o treinamento contínuo, enfatizando o aspecto cognitivo. Conclusão: A amostra pesquisada é uma população jovem, não inserida no mercado trabalhista, que não mostra relação entre a sua escolha e a especialidade da escola secundária onde se formou, incentivada pelos seus pais que geram e estimulam o interesse 
pelo ensino superior e demonstram adequação com alto comprometimento e solidariedade social, como expectativa profissional.

Palavras chave: Valores Sociais - Aluno - Orientação Vocacional.

\section{Introducción}

La orientación vocacional en la actualidad tiene por objetivo ayudar al sujeto a interrogarse sobre sus proyectos de vida, descubrir y desarrollar sus competencias, conocer diversas profesiones y formaciones, planificar sus trayectorias de estudio, trabajo, y en lo posible, prepararse para implementarlos, por esto es concebida como una instancia necesaria a lo largo de toda la vida (1). La elaboración de un proyecto vital, incluye una serie de elecciones sucesivas, forjadas sobre la base de cualidades saludables, direccionando objetivos, confianza, motivación, y expectativas hacia los nuevos trayectos. Afirma Casullo (2), que "la elaboración de un proyecto, forma parte del proceso de maduración afectiva e intelectual de las personas". Los conflictos y su resolución, las crisis vitales y los momentos de elección por los que transita el sujeto, están atravesados por la peculiaridad de su historia, por su subjetividad, por su situación socio-cultural, experiencias de aprendizaje, deseos y su novela familiar, que en suma, contribuyen a tejer las tramas de su futuro. La conformación del mismo se encuentra vinculada a la constitución de la identidad ocupacional, entendiendo a ésta como "la representación subjetiva de la inserción concreta en el mundo del trabajo, en el que puede autopercibirse incluido o excluido"(3). Implica además, la articulación del presente con la trayectoria pasada y las perspectivas futuras; como así también la posibilidad de integrar procesos afectivos e intelectuales que permitan una síntesis de las necesidades, expectativas y aspiraciones proyectadas en los contextos y escenarios imaginarios y reales en los que el sujeto se halla inserto. Da cuenta de la posibilidad de anticipar las diferentes representaciones, no sólo son las consecuencias de la socialización familiar, escolar y de la transmisión e internalización de actitudes y valores que conlleva, sino que son además, producidas activamente por cada sujeto. Cada joven elabora y desarrolla sus representaciones según la percepción que tiene de sus posibilidades para el futuro. La situación, requiere elaboración y consolidación de una identidad ocupacional, forma parte del proceso de maduración tanto afectiva como intelectual y supone un aprendizaje con relación al crecer (4). Beltrán Llavador (5) utiliza este término para referirse "a la posibilidad de establecer un horizonte no perceptible actualmente, pero al que se pretende llegar en un plazo prefijado". Asimismo, este autor realiza algunas consideraciones al respecto, supone trascender la inmediatez proyectando metas con el propósito de intervenir en la situación de partida, -representa la manifestación de intenciones y del sentido transformador de la realidad mediante lo que se proyecta, incluye la valoración y selección de estrategias y recursos a utilizar. Define Diana Aisenson (6), al "proyecto de vida como la actual representación de actividades y situaciones que se desarrollarán en el futuro, sea a corto, mediano o largo plazo". Es posible incluir en este concepto las expectativas, valores e intereses que llevan al sujeto a adoptar un modo de vida tanto en el área personal como familiar, social y ocupacional. En suma, estas son tareas que hacen a la posibilidad imaginaria de movilidad social y es necesario que el sujeto pueda llevarlas a cabo. "Elegir un estudio de nivel superior es una experiencia trascendente en la vida de una persona, porque comienza con esa decisión a proyectarse en el futuro. De esta manera, la carrera va articulando tres dimensiones: estudio- trabajo- y en un sentido más amplio, el tipo o estilo de vida que se llevará en la adultez"(7). La acción de elegir siempre estará marcada por circunstancias socio-históricas, políticas, económicas y culturales(2). Fortalecer la capacidad de los jóvenes para definir su proyecto de vida, dotarlos de los instrumentos básicos para que se conozcan y construyan su identidad, prepararlos para seguir estudiando, para ingresar al mundo laboral, son ejes centrales de las modalidades pedagógicas y orientaciones laborales vocacionales..."(8) por lo tanto prepararlos para 
el ingreso a la universidad es una realidad compleja que tiene que asumir la escuela, ya que intervienen múltiples dimensiones y se debe abordar en este nivel. Por otra parte, es necesario implementar estrategias integrales cuyo objetivo primordial sea el de buscar que los estudiantes se apropien de las capacidades, habilidades, conocimientos y estrategias necesarias que le permitan ingresar y permanecer en la universidad con un rendimiento académico que asegure su permanencia y una acomodación subjetiva que favorezca su desarrollo $(8,9)$. En tal sentido se destaca, que los sujetos que se encuentran en la transición entre "ser estudiante secundario" y "ser estudiante universitario" tienen escaso registro de lo que implica la Universidad, que con lógicas institucionales distintas a lo conocido por ellos, los enfrenta a un período de transición, un proceso de acomodación e integración que suele traer dificultades para la permanencia en el proyecto.

Lo social interviene de varias maneras: por medio del contexto concreto en que se sitúan los individuos y los grupos; a través de la comunicación que se establece entre ellos; de acuerdo con los marcos de aprehensión que proporciona su bagaje cultural; y mediante los códigos, valores e ideologías relacionadas con las posiciones y pertenencias sociales específicas (10). Se consideran los aportes de Denise Jodelet (11), que entiende la representación social "como una manera de interpretar y de pensar nuestra realidad cotidiana", es decir, una forma del conocimiento social. Correlativamente, es la actividad mental desplegada por individuos y grupos a fin de fijar su posición en relación con situaciones, acontecimientos, objetos y comunicaciones que les conciernen. La autora (11) plantea que, "la noción de representación social nos sitúa en el punto donde se interactúan lo psicológico y lo social". La representación social, designa también, una forma de conocimiento específico cuyos contenidos manifiestan la operación de procesos operativos y funcionales socialmente caracterizados; y en un sentido más amplio, designa una forma de pensamiento social.

En la actualidad, es fundamental proponer un sistema de intervención multidisciplinaria que permita la interacción de los distintos actores sociales que puedan ser importantes para el funcionamiento del área de orientación y necesarios para la educación de cada aspirante a una carrera universitaria. La orientación es una actividad inseparable del trayecto educativo del sujeto que lo debe acompañar de forma continua y sistemática. Planificada bajo esta perspectiva, la orientación puede resultar un proceso gradual, evolutivo, permanente y de efectos preventivos reales que colabora en la construcción del proyecto vital, en virtud de considerar que intervienen en la orientación vocacional tanto factores personales (intereses, aptitudes valores, motivaciones, expectativas y temores), como los factores sociales (el contexto familiar, contexto escolar, los pares, y el campo ocupacional) que, al ser reconocidos, favorece las decisiones autónomas, donde se integra el conocimiento de sí mismo y la realidad. Así, el proyecto vital, la transición y la inserción del sujeto al nivel universitario, son distintas aristas que conforman la identidad y contribuyen a su formación y consolidación (12).

Objetivo: Identificar las representaciones de los aspirantes de la carrera de Medicina con respecto al rol profesional.

\section{Métodología}

El presente trabajo es un estudio observacional y transversal, que se abordó metodológicamente, a partir de una encuesta implementada en el año 2007, a 2400 estudiantes que se preinscribieron para la carrera de Medicina. Del total de encuestas aplicadas, se obtuvieron 1857 registros completos que fueron seleccionados para el presente trabajo.

El instrumento utilizado estuvo conformado por 45 ítems, con opciones múltiples en sus respuestas, el cual fue anteriormente validado (13). En este trabajo se aborda la Dimensión Sociodemográfica, (ítems 1 a 22) la cual incluye las variables de identificación personal, edad, género, nacionalidad, lugar de nacimiento, estado civil, orientación de la especiali- 
dad de los estudios secundarios, carreras en las que se inscribe, si estudió otras carreras, relación laboral, si necesita trabajar para estudiar, qué trabajo le gustaría realizar en la actualidad y en qué ámbito, qué actividades realiza en su tiempo libre, con quién vive, edad, ocupación y formación de los padres. La Dimensión Proyecto de vida (personal, profesional, laboral) y la imagen del rol, comprenden desde los ítems 41 al ítems 45 y en ellos se abordan aspectos como, la explicitación de la imagen del rol profesional de la carrera elegida, qué conoce del campo laboral, la especialidad en que le gustaría desempeñarse, las características y cualidades personales que debe poseer o desarrollar el profesional de la carrera que eligió y qué proyectos tiene como futuro profesional de la salud.

Análisis estadístico de los datos: se efectuó un análisis univariado para describir la distribución y frecuencia de presentación de cada variable incluida en el estudio. Posteriormente, se efectuó un análisis bivariado de asociación entre las variables utilizando el test de $\chi^{2}$ ajustado o se aplicó ANOVA según corresponda. Se trabajó con un nivel de confianza del 95\% utilizando el software estadístico InfoStat.

En todos los casos se especificó el resguardo ético de los datos suministrados por los participantes.

\section{Resultados}

En cuanto a la Dimensión Sociodemográfica se observó que la distribución por sexo fue $62 \%$ de mujeres y $38 \%$ de varones, con una media de edad de $19,03 \pm 0,06$ años, siendo la media de edad de las mujeres menor que la de los varones (18,94 años y 19,19 años, respectivamente $\mathrm{p}=0,03)$. Respecto al estado civil el $98 \%$ son solteros. Al analizar la nacionalidad se observó que el $98 \%$ eran de nacionalidad argentina, el $2 \%$ extranjeros/as. Se observó que el $56 \%$ de la población procede de Córdoba y el $42 \%$ al resto del país, en donde el $17 \%$ corresponde al Noroeste, el $17 \%$ Noreste y el $8 \%$ proceden da la zona sur del territorio argentino. La distribución de los estudiantes de acuerdo a las áreas de la Especialidad del Secundario reveló un predominio en la Especialidad en Ciencias Naturales (respecto a las otras especialidades) $(\mathrm{p}<0,001)$. Cuando se analizó esta frecuencia de la orientación de los estudios del nivel medio, agrupado en relación al sexo se detectó que las mujeres pertenecen con mayor frecuencia al área de las Ciencias Naturales que los varones $(\mathrm{p}=0,03)$. El 84\% refiere no haber cursado otra carrera, y el $16 \%$ restante cursó en las áreas de Ciencias de la Salud, Ciencias Exactas y Ciencias Económicas. El $91 \%$ de los encuestados no trabaja, y al indagar acerca de la elección laboral el 23\% le gustaría realizar trabajos en relación a Ciencias de la Salud. En relación a las actividades que realizan en su tiempo libre se expresa un predominio de las actividades deportivas $(\mathrm{p}=0,0034)$.Acerca de la ocupación y de la formación profesional del padre, se evidencia el predominio de actividades en áreas no relacionadas con la salud, el $15 \%$ realiza actividades en áreas relacionadas a la salud, de los cuales sólo el 12\% son Médicos. Acerca de la ocupación y formación profesional de las madres de los estudiantes, el $86 \%$ trabaja en áreas no relacionadas a la salud, un 14\% trabaja en relación al área de salud, de los cuales sólo el 5\% son Médicas.

Con respecto al Ítem 41 "explicite la imagen del Rol Profesional de la carrera elegida, ¿Cómo se imagina en el rol?”, se observa las categorías cualidades personales aspectos, preventivos de la carrera, rol y especialidad (Figura 1). 


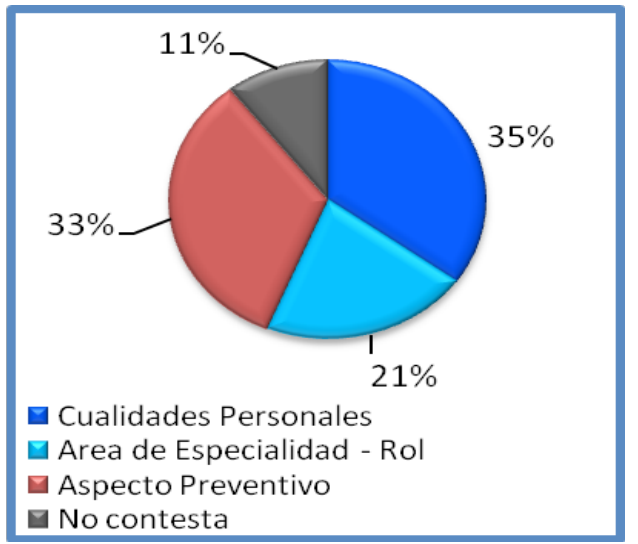

Figura $\mathbf{N}^{\circ}$ 1: Distribución de las respuestas de los estudiantes preinscriptos a la carrera de Medicina de la Facultad de Ciencias Médicas, con respecto a las explicitación de la imagen del rol profesional.

En la Figura 2 se observa la distribución de respuestas en relación al ítem 42, "Del campo laboral de la carrera ¿qué conoce?”, con predominio al conocimiento en el campo de las especialidades médicas $(\mathrm{p}<0,02)$.

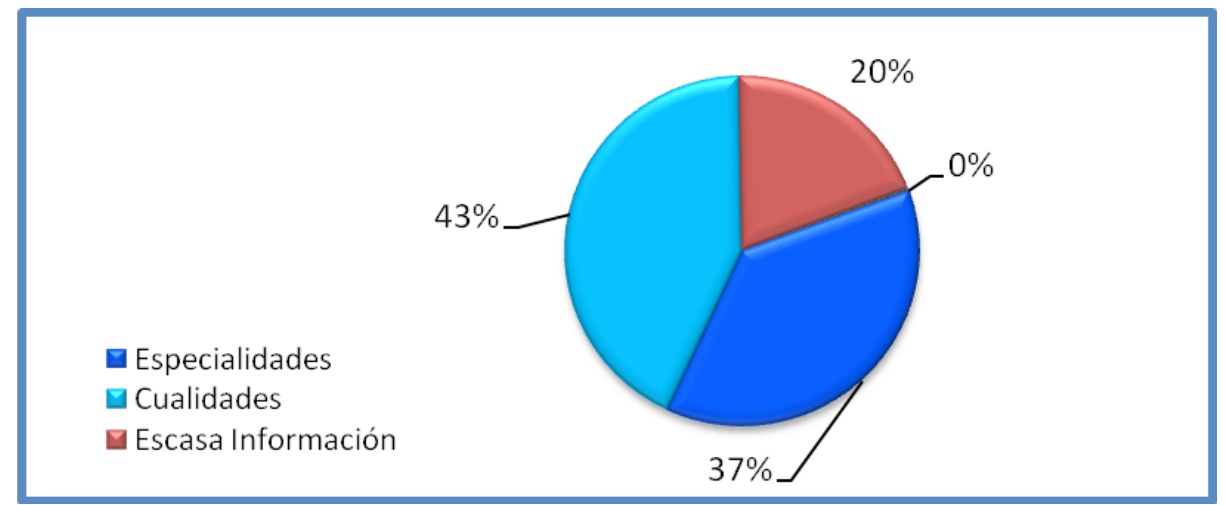

Figura $\mathbf{N}^{\circ}$ 2: Distribución de las respuestas de los estudiantes preinscriptos a la carrera de Medicina de la Facultad de Ciencias Médicas, con respecto a lo que conoce del campo laboral.

El ítem 43, "en qué especialidad le gustaría desempeñarse como futuro profesional”, plantea la posibilidad de responder en primer y segundo lugar. Se observa el área de las Especialidades Médicas como área elegida en primer y segundo lugar $(\mathrm{p}<0,02)$. En el área de Especialidades Médicas se incluyen todas las áreas de la Medicina a excepción del área Quirúrgica, área Clínica y el área Medicina Familiar. (Figura 3). 


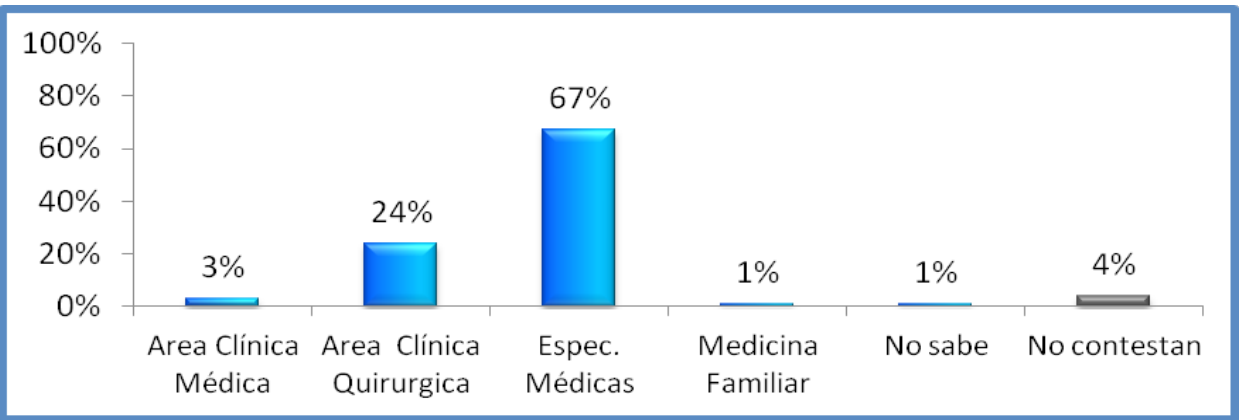

Figura $\mathbf{N}^{\circ}$ 3: Distribución de las respuestas de los estudiantes preinscriptos a la carrera de Medicina de la Facultad de Ciencias Médicas, con respecto a la especialidad en que le gustaría desempeñarse como primera opción.

El ítem 44, ¿Qué características y cualidades personales debe poseer o desarrollar el profesional de la carrera que eligió?,

En las características que debe poseer o desarrollar el profesional mencionadas en primer lugar, predominan las cualidades personales $(\mathrm{p}<0,02)$, seguido por la formación permanente y la responsabilidad (Figura 4).

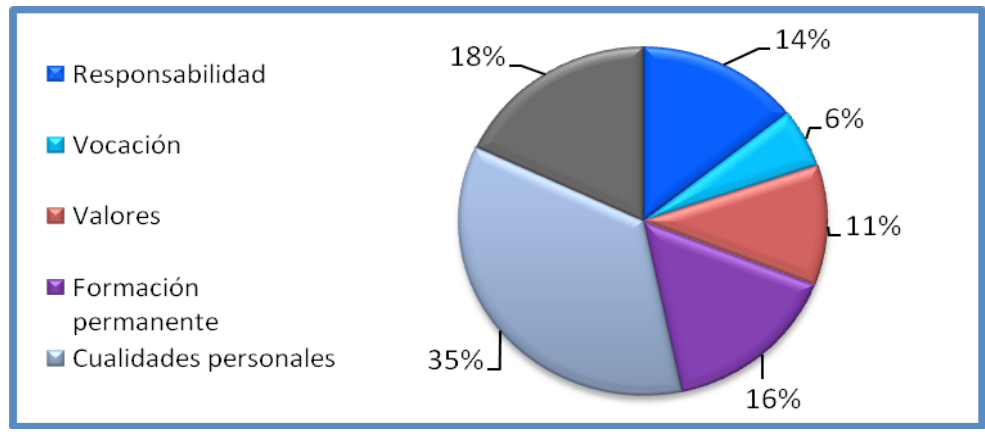

Figura $N^{\circ}$ 4: Distribución de las respuestas de los estudiantes preinscriptos a la carrera de Medicina de la Facultad de Ciencias Médicas, con respecto a qué características y cualidades debe poseer o desarrollar el profesional de la carrera, en primer lugar.

Con respecto al ítem 45, "Como futuro profesional de la salud ¿qué se ve haciendo de acá a 7 años?" Los resultados muestran porcentajes similares entre el compromiso social (ayudar a la gente, actuar preventivamente, reafirmando el aspecto social y solidario del Rol Profesional) y la idoneidad profesional (continuar con la formación permanente), (Figura 5). 


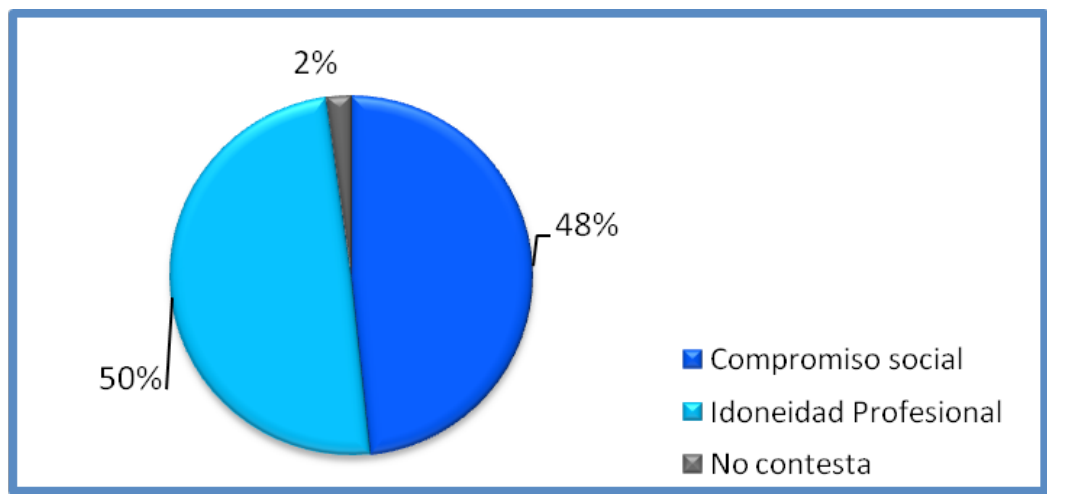

Figura $\mathbf{N}^{\circ}$ 5: Distribución de las respuestas de los estudiantes preinscriptos a la carrera de Medicina de la Facultad de Ciencias Médicas, con respecto al proyecto del rol profesional dentro de 7 años.

\section{Discusión}

Con respecto a la distribución de los estudiantes según el sexo, se observó una mayor proporción de mujeres en relación a los varones, lo cual coincide con los datos referenciados en trabajos anteriores, respecto a la distribución de los estudiantes en relación al género en el ámbito de la carrera de Medicina de la Universidad Nacional de Córdoba (14). El predominio de mujeres entre los estudiantes hallado en el presente estudio, también acuerda con investigaciones realizadas en otros países como en la Universidad de México y Barcelona $(15,16)$. En referencia a la ocupación de los padres, se presenta un predominio laboral en áreas no relacionadas a la salud. Referido a esta última característica, cabe destacar la no coincidencia de la profesión de los padres con la elección de los estudiantes a la carrera de Medicina, lo cual no acuerda con el imaginario social respecto a esta temática, ni tampoco con la investigaciones que abordan este tema tales como la investigación de la Universidad del Noreste (17), que hace mención a este aspecto. Referido a la misma temática, una investigación realizada en Perú, sostiene que tener un familiar médico o tener un familiar profesional de la salud, o una buena experiencia con un médico incrementa la motivación hacia la elección de la carrera, o bien por el contrario asumen que la frecuencia de la elección de la carrera de Medicina en hijos varones de médicos, no es significativa (18). Por otra parte, la buena imagen del rol profesional juega un papel favorable en la recepción de la profesión, en este sentido la cercanía de un modelo médico durante la niñez y adolescencia facilitaría la identificación y conocimiento de la posición social, estimulando a su vez, la afinidad por tal profesión a más temprana edad (19). Por ello, las influencias conflictivas o negativas con el rol podrían desfavorecer dicha elección. De manera general, esto explicaría por qué la presencia de un familiar profesional de la salud y la buena experiencia con un médico se encuentran asociadas con la elección de la carrera de Medicina, hallazgo descripto también por otros autores. Estas investigaciones realizadas en Perú, sostienen la incidencia familiar, o una buena experiencia previa con un médico, puede influir en la elección positivamente. En relación a la imagen del rol profesional se ha considerado que cada estudiante accede a la universidad con representaciones construidas acerca de las profesiones, los profesionales y las carreras, a partir de las propiedades que objetivamente los caracterizan, aunque también conllevan un carácter significante. En su construcción intervienen la subjetividad, las percepciones y valoraciones que son el resultado de toda la experiencia vital previa de los sujetos. Cada uno además, enriquece esas representaciones con la información y descripciones que obtienen en los procesos de interacción y comunicación social como de los medios de comunicación, de los padres y 
amigos. Por su naturaleza simbólica y social, las representaciones implican valoraciones y expectativas que influyen en el tipo y calidad de la relación que se mantiene con el objeto representado, en este caso la carrera de Medicina (19).

Con respecto a las cualidades y características necesarias a sostener en el desarrollo del rol médico, los estudiantes manifestaron que el compromiso social y la formación permanente son los aspectos más importantes a mantener en la elección y durante el desarrollo de la carrera. Investigaciones como la de los estudiantes de Medicina de la Pontificia Universidad Católica de Chile (20) muestran una percepción del médico esperado que incluye tanto características humanitarias como científicas. En dicha investigación se hace referencia a que en todos los grupos aparecen ideales que debiera poseer el médico, afirman que los estudiantes mantienen ciertos ideales hasta el fin de la carrera, sin embargo, también se ha informado que con el avance en sus estudios, pierden la valorización de los ideales con los que ingresan. Por otro lado, señalan que les cuesta integrar valores como altruismo y servicio a su práctica cotidiana.

La experiencia indica que la participación activa de los jóvenes para determinar qué atributos son necesarios para un buen médico, es una forma positiva de asegurar que abrazan la importancia de tales cualidades y atributos en sí mismos (19).

Otras investigaciones sostienen, como la Dirección de Estadística e Informática de la Asamblea Nacional de Rectores (Lima, Perú), que la decisión del futuro profesional parece depender de múltiples factores. Al respecto, Zuckerman (21) considera que esta decisión depende principalmente de la relación de tres factores: los sociodemográficos, estructurales o institucionales y los de personalidad. Fernández y López (16) describen que en la relación participan los factores sociales, vocacionales y estructurales. Por su parte, Jarrillo y colaboradores (22) encuentran tres determinantes principales en su modelo del comportamiento en torno a una profesión: los procesos educativos, los elementos de socialización cultural y una dimensión familiar.

\section{Conclusiónes}

En la presente investigación se destaca que la población en estudio, es una población joven, no inserta en el mercado laboral, que no muestra relación entre su elección y la especialidad del secundario que ha cursado, y se infiere que estos jóvenes son altamente estimulados por sus progenitores quienes les generan y alientan interés por realizar estudios superiores. Además de mostrar como expectativas profesionales, la idoneidad con alto compromiso y solidaridad social. La carrera universitaria, representa para el sujeto, un movimiento subjetivo y extrínseco, por los cambios que implica este pasaje en cuanto a la organización de los procesos internos que se configuran de diferentes formas en el sujeto y en la personalidad, así como en los diversos espacios sociales en los que está inserto. Se espera que estos procesos, se configuren en las propias trayectorias de los estudiantes, a través de las estrategias personales, sociales e institucionales que le permitirán no solo permanecer en la carrera sino prepararse para el rol profesional (23).

\section{Bibliografía}

1. Aisenson, D. y Otros; Después de la escuela, Transición, construcción de proyectos, trayectorias e identidades de los jóvenes. Buenos Aires. Ed. Eudeba; 2002.

2. Casullo, M. M., Cayssials, A.N. "Proyecto de vida y decisión vocacional". Paidós. Buenos Aires .2006.

3. Módulo Bibliográfico de la Cátedra de Orientación Vocacional y Ocupacional. Facultad de Psicología. Universidad Nacional de Córdoba. Argentina. 2019.

4. Gavilán, M. "La transformación de la Orientación Vocacional. Hacia un nuevo paradigma”. Lugar Editorial. Buenos Aires. 2017. 
5. Beltrán LLavador, F; Diseñar la coherencia escolar. Madrid. Ed. Morata. 2000.

6. Aisenson, D; La Orientación Vocacional como dispositivo articulador entre educación, trabajo y salud. Buenos Aires. Ed. Eudeba. 1997.

7. Rascovan, S. Las elecciones vocacionales de los jóvenes escolarizados. Proyectos, expectativas y obstáculos. Noveduc. Buenos Aires. 2010.

8. Pássera, J. Comp. Orientación Vocacional. Una propuesta teórico-práctica. Córdoba. Ed. Brujas. 2014.

9. Stábile, C. El departamento de orientación en la escuela. En J Pássera (Comp); Orientación vocacional. Una propuesta teórico-práctica (pp. 65-68). Córdoba: Brujas. 2014.

10. Aisenson D; Castorina J; Elichiry N; Lenzi A; Schelemenson S. Aprendizajes, sujetos y escenarios. Ed. Oveduc.BsAs.2007

11. Jodelet, D; "La representación social: fenómenos, conceptos y teorías", en Moscovici, S. (comp.).Psicología Social. Pensamiento y vida social. Psicología Social y problemas sociales. Cap.13. Barcelona. Ed. Paidós. 1999.

12. Guichard, J; "Los dos pilares de las prácticas en orientación, fundamentos conceptúales y finalidades sociales" en después de la escuela. Transición, construcción, de proyectos. Buenos Aires. Ed. Eudeba. 2002.

13. Stabile C.; Tesis doctoral, “Análisis de los factores interactuantes de la problemática vocacional abordada desde la promoción de la salud". Facultad de Ciencias Médicas. Universidad Nacional de Córdoba. Argentina.2012.

14. Antuña A, Fernández,R.-Investigación en educación Médica: Análisis de la Propuesta Metodológica para la Admisión en la Facultad de Ciencias Médicas -Universidad Nacional de Córdoba .Argentina. 2007

15. Eloína Castro Lara,M.D.N. Hugo E. Hernández Carrasco. Profesionistas Asociados para la Comunicación y Salud de Puebla, Representaciones Sociales en torno a los Médicos: Construcción identitaria durante su formación. -Mexico2014.

16. López Rupérez F .La Gestión de calidad en Educación. Madrid. España. Ed. La Muralla 89-106. 1994.

17. Said Rücker PB, Chiapello JA, Espíndola de Markowsky ME. Evaluación del desempeño intelectual de los alumnos que se postulan para ingresar a la Carrera de Medicina. Revista de Postgrado de Ia Cátedra de Medicina, 126: 4-9. 2003.

18. García JC. La educación médica en América Latina. Washington D.C.: OPS; Publicación Científica N²25. Rev. Medica, Hered. 2004

19. Mine Sehiralti, Aslihan Akpinar, Nermin Ersoy. Attributes of a good physician: what are the opinions of first-year medical students? J Med Ethics; 36: 121-125 doi:10.1136/jme.2009.032854. 2010.

20. García Huidobro M, Diego et al. Expectativas de estudiantes de Medicina de pregrado en relación al perfil de médico esperado. Rev. méd. Chile, vol.134, n.8 [cited 2010-12-13], pp. 947-954. 2006.

21. Zuckerman H. Structural factors as determinants of career patterns in medicine. $\mathbf{J}$ Med Educ; 53: 453-63. 1998.

22. Jarrillo-Soto E, Arroyave MG, Chapela MC. Algunos determinantes en la conformación de los patrones profesionales en estudiantes de Medicina. Salud Pública México; 34: 455-66. 2000.

23. Clark,C.yStábile,C.“Acompañamiento a las trayectorias académicas estudiantiles. Dirección de Inclusión y Ciudadanía Estudiantil. Secretaria de Asuntos Estudiantiles, Universidad Nacional de Córdoba. Argentina.2016. 